\title{
Luminous and Obscured Quasars and Their Host Galaxies
}

\author{
Agnese Del Moro ${ }^{*}$, David M. Alexander ${ }^{2}$, Franz E. Bauer ${ }^{3,4,5,6}$, Emanuele Daddi ${ }^{7}$, \\ Dale D. Kocevski ${ }^{8}$, Flora Stanley ${ }^{9}$ and Daniel H. Mclntosh ${ }^{10}$ \\ ${ }^{1}$ Max-Planck-Institut für Extraterrestrische Physik, Garching, Germany, ${ }^{2}$ Department of Physics, Centre for Extragalactic \\ Astronomy, Durham University, Durham, United Kingdom, ${ }^{3}$ Facultad de Física, Instituto de Astrofísica, Pontificia Universidad \\ Católica de Chile, Santiago, Chile, ${ }^{4}$ Millennium Institute of Astrophysics (MAS), Santiago, Chile, ${ }^{5}$ EMBIGGEN Anillo, \\ Concepción, Chile, ${ }^{6}$ Space Science Institute, Boulder, CO, United States, ${ }^{7}$ CEA, IRFU, DAp, AIM, Université Paris-Saclay, \\ Université Paris Diderot, Sorbonne Paris Cité, CNRS, Gif-sur-Yvette, France, ${ }^{8}$ Department of Physics and Astronomy, \\ University of Kentucky, Lexington, KY, United States, ${ }^{9}$ Department of Space Earth and Environment, Chalmers University of \\ Technology, Onsala Space Observatory, Onsala, Sweden, ${ }^{10}$ Department of Physics and Astronomy, University of \\ Missouri-Kansas City, Kansas City, MO, United States
}

\section{OPEN ACCESS}

Edited by:

Ascensión Del Olmo,

Instituto de Astrofísica de Andalucía (CSIC), Spain

Reviewed by: Lorena Hernandez-Garcia, University of Valparaíso, Chile

Begoña Garcia-Lorenzo, Instituto de Astrofísica de Canarias,

Spain

${ }^{*}$ Correspondence:

Agnese Del Moro adelmoro@mpe.mpg.de

Specialty section:

This article was submitted to

Cosmology,

a section of the journal

Frontiers in Astronomy and Space

Sciences

Received: 30 October 2017 Accepted: 18 December 2017 Published: 12 January 2018

Citation: Del Moro A, Alexander DM, Bauer FE, Daddi E, Kocevski DD, Stanley $F$ and McIntosh DH (2018) Luminous and Obscured Quasars and Their Host Galaxies.

Front. Astron. Space Sci. 4:67. doi: 10.3389/fspas.2017.00067
The most heavily-obscured, luminous quasars might represent a specific phase of the evolution of the actively accreting supermassive black holes and their host galaxies, possibly related to mergers. We investigated a sample of the most luminous quasars at $z \approx 1-3$ in the GOODS fields, selected in the mid-infrared band through detailed spectral energy distribution (SED) decomposition. The vast majority of these quasars ( $80 \%)$ are obscured in the X-ray band and $\sim 30 \%$ of them to such an extent, that they are undetected in some of the deepest (2 and $4 \mathrm{Ms}$ ) Chandra X-ray data. Although no clear relation is found between the star-formation rate of the host galaxies and the X-ray obscuration, we find a higher incidence of heavily-obscured quasars in disturbed/merging galaxies compared to the unobscured ones, thus possibly representing an earlier stage of evolution, after which the system is relaxing and becoming unobscured.

Keywords: galaxies: active, quasars: general, quasars: supermassive black holes, X-rays: galaxies, infrared: galaxies, galaxies: star formation

\section{INTRODUCTION}

The similarity between the accretion history of galaxies and supermassive black holes (SMBHs), peaking at redshift $z \approx 1-2$ (e.g., Madau et al., 1996; Hopkins et al., 2006; Brandt and Alexander, 2015), suggests that there is a connection between the evolution of a galaxy and the black hole in their center. Such connection has also been hinted by the observed correlations between the BH mass and the velocity dispersion of the stars in the bulge $\left(M_{\mathrm{BH}}-\sigma\right.$ relation; Ferrarese and Merritt, 2000; Gebhardt et al., 2000) or with the bulge mass $\left(M_{\mathrm{BH}}-M_{\text {bulge }}\right.$; Kormendy and Richstone, 1995; Magorrian et al., 1998). Whether this parallel evolution is simply due to a larger gas supply at high redshift, feeding both the SMBH and star formation (SF), or whether there are other processes selfregulating the $\mathrm{SMBH}$ and galaxy growth (e.g., AGN feedback) is still uncertain (e.g., Alexander and Hickox, 2012; Kormendy and Ho, 2013).

Studying active galactic nuclei (AGN) at all cosmic epochs is crucial to fully understand the accretion history of the SMBHs and their role in galaxy evolution. However, most of the accretion onto SMBHs is expected to be heavily obscured by dust and gas, making the identification of the most obscured AGN population very challenging, even in the deepest X-ray surveys. According to the unified model (e.g., Antonucci, 1993), AGN appear obscured due to orientation effects, 
when our line-of-sight crosses high column densities $\left(N_{\mathrm{H}}\right)$ of circumnuclear material, the so called "torus". However, it has been suggested that the most heavily-obscured, Compton-thick (CT; where $N_{\mathrm{H}}>1.5 \times 10^{24} \mathrm{~cm}^{-2}$ ) AGN, especially the (intrinsically) most luminous ones, could represent a particular phase of galaxy evolution, associated to a major merger, when a lot of gas and dust are funneled into the center of the galaxy, deeply hiding the active nucleus within it (Di Matteo et al., 2005; Hopkins et al., 2006; see also Alexander and Hickox, 2012, for a review). Yet, the emission reprocessed by the obscuring dust is re-emitted in the mid-infrared (MIR) band, which can therefore be used to find even the most obscured and elusive quasars.

\section{SAMPLE SELECTION}

The sample was selected from a large catalog of $24 \mu \mathrm{m}$-detected sources within the GOODS-Herschel North and South fields. We performed detailed SED decomposition using Spitzer 8, 16, and $24 \mu \mathrm{m}$ and Herschel 100,160, and $250 \mu \mathrm{m}$ data, to separate the AGN from SF emission. We adopted the AGN and star-forming galaxy (SFG) templates described in Mullaney et al. (2011) and Del Moro et al. (2013); details of the SED fitting are described in Del Moro et al. (2016). Amongst these sources we selected the most luminous quasars in the MIR band, with rest-frame $6 \mu \mathrm{m}$ luminosity of $\nu \mathrm{L}_{\mathrm{AGN}, 6 \mu \mathrm{m}}>6 \times 10^{44} \mathrm{erg} \mathrm{s}^{-1}$, corrected for the galaxy contribution, at redshift $z=1-3$. This selection results in a sample of 33 sources.

\section{ANALYSES AND RESULTS}

\subsection{AGN: Heavily Obscured Population at $z \approx 2$}

To characterize the quasars in our sample, we used the deep Xray Chandra data available in the Chandra Deep Field North (CDF-N; 2 Ms; Alexander et al., 2003) and Chandra Deep Field South (CDF-S 4 Ms; Xue et al., 2011). For details on the data reduction we refer to Alexander et al. (2003), Luo et al. (2008), and Xue et al. (2011). Of our 33 quasars, $24(\sim 73 \%)$ are detected in the X-ray band, while $9(\sim 27 \%)$ remain undetected, despite being intrinsically very luminous in the MIR band. These sources are candidates to be the most heavily obscured, CT AGN.

For the sources that are detected in the X-rays, we extracted the spectra using ACIS Extract (AE; Broos et al., 2010, 2012) and analyzed them using a simple absorbed power-law model (including Galactic and intrinsic absorption) to constrain the amount of $N_{\mathrm{H}}$. In Figure 1 we show the $N_{\mathrm{H}}$ distribution of the sample. We find that the majority of these quasars $(16 / 24 ; \sim 67 \%)$ are obscured by columns of $N_{\mathrm{H}}>10^{22} \mathrm{~cm}^{-2}$, of which more than half $(9 / 16)$ are heavily obscured $\left(N_{\mathrm{H}}>2 \times 10^{23} \mathrm{~cm}^{-2}\right)$. Amongst these heavily-obscured sources, we identified six of them as CT quasars from the X-ray spectral analysis, using spectral models appropriate for heavily-obscured sources, such as, PLCABS (Yaqoob, 1997) and TORUS (Brightman and Nandra, 2011). The fraction of obscured quasars in our sample reaches $\sim 76 \%$, and $54 \%$ of heavily-obscured quasars, if we include

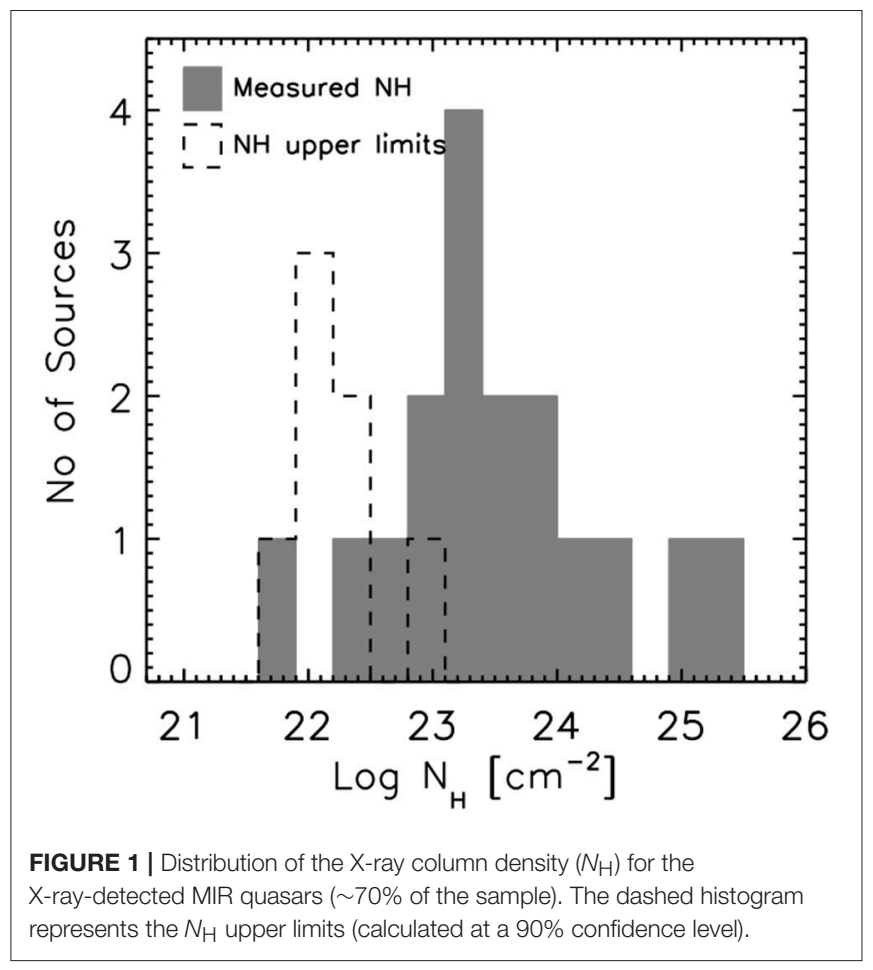

the X-ray-undetected sources, assuming these are the most heavily CT ones. Indeed, the comparison between the intrinsic luminosity at $6 \mu \mathrm{m}$ and the X-ray luminosity upper limit of these sources suggests that the X-ray emission is heavily suppressed compared to the intrinsic $L_{X}-L_{6} \mu \mathrm{m}$ relation found for AGN (e.g., Lutz et al., 2004; Fiore et al., 2009; Gandhi et al., 2009), making them very good candidates to be heavily CT quasars.

We note that amongst the X-ray undetected quasars in the sample, there is one source, \#28 (see Table 1 from Del Moro et al., 2016), that is now detected in the 7 Ms CDF-S catalog (XID 28; Luo et al., 2017). This source has a very flat effective photon index of $\Gamma<0.93$ (compared to the typical $\Gamma \approx 1.8$ for unabsorbed $\mathrm{AGN}$ ) and an extremely low rest-frame X-ray luminosity $\left(L_{0.5-7 \mathrm{keV}} \approx 1.5 \times 10^{41} \mathrm{erg} \mathrm{s}^{-1}\right.$, uncorrected for absorption) compared to its intrinsic luminosity measured in the MIR band ( $\left.\log v \mathrm{~L}_{6} \mu \mathrm{m}=45.97 \mathrm{erg} \mathrm{s}^{-1}\right)$, consistent with the upper limit reported in our analysis (Del Moro et al., 2016) ${ }^{1}$, supporting our assumption that the source might be a heavily obscured, CT quasar.

These results suggest that there is a large population of heavily-obscured, intrinsically luminous quasars at high redshift, which are very elusive even for deep X-ray surveys. These sources might constitute a special phase of the $\mathrm{BH}$-galaxy evolution, where the actively growing $\mathrm{BH}$ is embedded in large amounts of gas and dust, possibly as a result of a recent merger.

\footnotetext{
${ }^{1}$ We note however, that the photometric redshift assumed in our analysis of this source $(z=2.55)$ differs from that reported in the Luo et al. (2017) catalog $(z=1.81)$.
} 


\subsection{AGN Host Galaxies: Star-Formation Rates and Merger Fraction}

To study the characteristics of the host galaxies of these MIRluminous quasars, we investigated their star-formation rates (SFRs) and their morphologies, in particular the disturbance or distortion of their morphology, as an indication of galaxy mergers and interactions. We derived the SFR of each galaxy from its farinfrared (FIR) luminosity (or upper limit) resulting from the SED decomposition in the IR band (see section 2 and Del Moro et al., 2016), assuming a Salpeter initial mass function (Salpeter, 1955) and the relation from Kennicutt (1998). We also calculated the average SFR separately for the unobscured/moderately obscured quasars $\left(N_{\mathrm{H}}<2 \times 10^{23} \mathrm{~cm}^{-2}\right)$ and for the heavily-obscured quasars $\left(N_{\mathrm{H}}>2 \times 10^{23} \mathrm{~cm}^{-2}\right)$ in three different redshift bins (see Figure 2). To estimate the average SFRs accounting for the upper limits, we used the Kaplan-Meier (KM) product limit estimator (Kaplan and Meier, 1958), a non-parametric maximum-likelihood estimator of the distribution function (see Stanley et al., 2015, for details on the method).

We find that the average SFR increases with redshift, in general agreement with the SFR main sequence of galaxies (Figure 2). Moreover, although the heavily-obscured sources seem to have a slightly enhanced SFR compared to the unobscured/moderately obscured ones (especially at $z \approx 2$ ), these differences are not statistically significant, and therefore we find no clear dependence of the amount of SF in the galaxy with the X-ray obscuration of the quasar. This suggests that the obscuration in the X-ray band

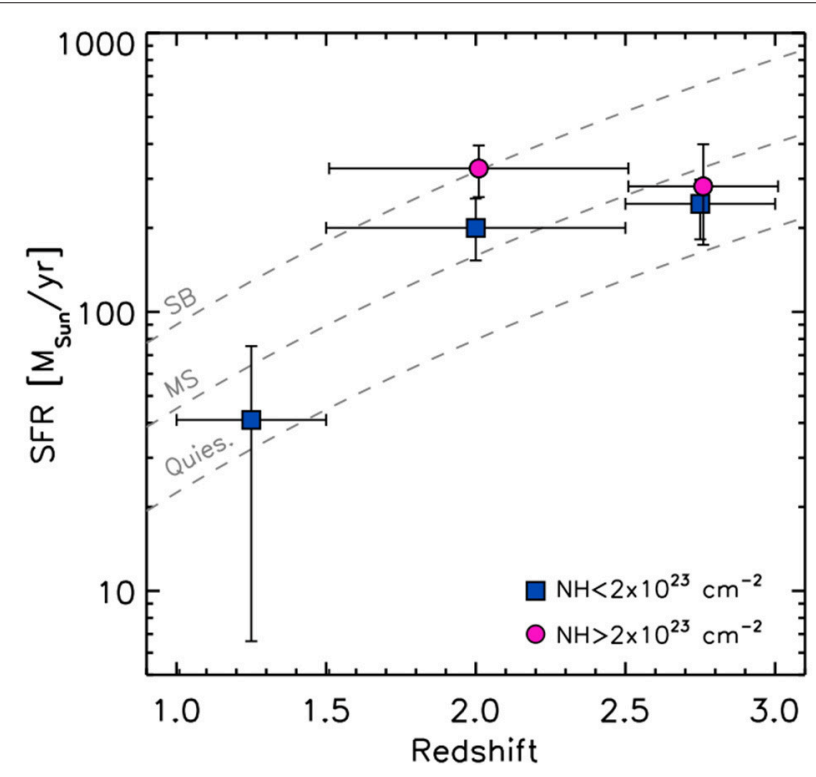

FIGURE 2 | Average star-formation rate (SFR) for the

unobscured/moderately-obscured quasars $\left(N_{H}<2 \times 10^{23} \mathrm{~cm}^{-2}\right.$; blue squares) and the heavily-obscured quasars $\left(N_{H}>2 \times 10^{23} \mathrm{~cm}^{-2}\right.$; magenta circles) in our sample, divided in three redshift bins. None of heavily-obscured quasars are detected in the lowest redshift bin. The gray dashed lines represent the SFR track for main-sequence $(M S)$, starburst $(S B$; SFR $M S \times 2)$ and quiescent (Quies.; SFR ${ }_{M S} / 2$ ) galaxies with a typical mass of $M_{*}=9 \times 10^{10} M_{\odot}$ (e.g., Elbaz et al., 2011). is likely confined in the nuclear regions and not related to the presence of gas on larger scales (e.g., Rosario et al., 2012; Rovilos et al., 2012).

Using the high-resolution optical HST images available in the GOODS-N and GOODS-S fields, as part of the GOODS and CANDELS projects (Giavalisco et al., 2004; Grogin et al., 2011), we visually inspected the morphology of these galaxies to identify signs of distortions or disturbances, which would indicate a recent galaxy merger/interaction event. We adopted a similar classification scheme to that used by Kocevski et al. (2012); see Del Moro et al. (2016, for details), separating the sources into "disturbed" and "undisturbed". In Figure 3 we show the fraction of sources having disturbed and undisturbed morphologies over the total, dividing them again into unobscured/moderately obscured (blue squares) and heavily obscured (magenta circles), as in Figure 2. We find that a relatively high fraction of our sources shows signs of distortions/interactions $(\approx 40 \%)$, higher than those typically found at low redshift $(\sim 15-20 \%$ at $z<$ 1; e.g., Cisternas et al., 2011). This is in agreement with the trend of increasing major-merger fraction with redshift seen in previous works (e.g., Conselice et al., 2003; Treister and Urry, 2006; Kartaltepe et al., 2007). We find that, on average, the most-heavily obscured quasars tend to have more disturbed morphologies than the unobscured/moderately obscured ones ( $\approx 53$ vs. $\approx 20 \%$, respectively); although the errors on these fractions are large due to the small number of sources in our sample, the difference between the two quasar populations is

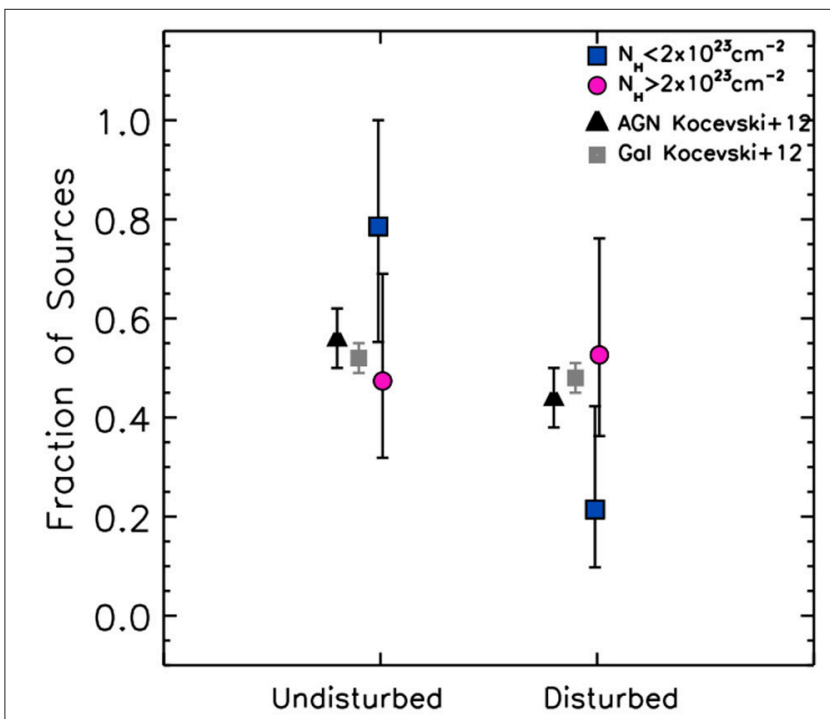

FIGURE 3 | Fraction of MIR quasar hosts showing disturbed and undisturbed galaxy morphologies, classified using HST images. The fractions and $1 \sigma$ uncertainties for the unobscured/moderately-obscured quasar hosts $\left(N_{\mathrm{H}}<2 \times 10^{23} \mathrm{~cm}^{-2}\right)$ are plotted as blue squares and for the heavily-obscured quasars $\left(\mathrm{N}_{\mathrm{H}} \geq 2 \times 10^{23} \mathrm{~cm}^{-2}\right)$ as magenta circles. For comparison we also show the fractions for $z \approx 2$ AGN and non-AGN samples from Kocevski et al. (2012): filled black triangles and gray squares, respectively. The unobscured/moderately-obscured quasars reside preferentially in undisturbed systems, while the heavily-obscured quasars are equally found in disturbed and undisturbed systems. 
significant at the $90 \%$ confidence level (Fisher exact probability test: $P=0.087$ ). This trend is seen also in other studies (e.g., Kocevski et al., 2015; Ricci et al., 2017). We note, however, that these studies investigated samples in different redshift and/or luminosity ranges compared to ours, and therefore the actual fractions of sources with disturbed morphologies are not directly comparable. The smaller fraction of disturbed systems we found for the unobscured/moderately-obscured quasars could be interpreted within the SMBH-galaxy evolutionary models, as the unobscured quasars would represent a later stage of the evolution compared to the heavily-obscured sources and the distortion features due to mergers or interactions might have faded by the time these quasars are observed as unobscured, given the relaxation time of a galaxy is typically 200-400 Myr (e.g., Lotz et al., 2010). On the other hand, for the most heavily-obscured quasars, which might represent a younger stage of evolution after a merger in this scenario, the signatures of the recent interactions are still evident in their hosts.

\section{CONCLUSIONS}

We have investigated the AGN and host galaxy properties of a sample of 33 quasars at $z=1-3$ within the GOODS-Herschel fields, selected in the MIR band through detailed SED analysis to have an intrinsic AGN luminosity of $\nu \mathrm{L}_{6 \mu \mathrm{m}}>6 \times 10^{44} \mathrm{erg} \mathrm{s}^{-1}$. Despite being intrinsically the most luminous quasars within these fields, $\sim 26 \%$ of them are not detected in the deep 2 and 4 Ms Chandra X-ray data covering these sky areas.

We performed X-ray spectral analysis of the $24 \mathrm{X}$-ray-detected sources to investigate the AGN properties, and we found that the vast majority $(\sim 67 \% ; 16 / 24$ sources $)$ are obscured by $N_{\mathrm{H}}>$ $10^{22} \mathrm{~cm}^{-2}$, with more than half of them (9/16) being heavily obscured $\left(N_{\mathrm{H}}>2 \times 10^{23} \mathrm{~cm}^{-2}\right)$. Including the X-ray undetected sources, which are likely to be the most heavily CT AGN, these fractions reach $\sim 76 \%$ ( $\sim 54 \%$ are heavily obscured). This means that there is a very large population of heavily obscured, intrinsically luminous quasars at redshift $z \approx 2$, which can be revealed in the IR band, but remains (in part) undetected in the $\mathrm{X}$-ray band.

\section{REFERENCES}

Alexander, D. M., Bauer, F. E., Brandt, W. N., Schneider, D. P., Hornschemeier, A. E., Vignali, C., et al. (2003). The chandra deep field north survey. XIII. 2 Ms point-source catalogs. Astron. J. 126, 539-574. doi: 10.1086/376473

Alexander, D. M., and Hickox, R. C. (2012). What drives the growth of black holes? New A Rev. 56, 93-121. doi: 10.1016/j.newar.2011.11.003

Antonucci, R. (1993). Unified models for active galactic nuclei and quasars. Annu. Rev. Astron. Astrophys. 31, 473-521. doi: 10.1146/annurev.aa.31.090193.002353

Brandt, W. N., and Alexander, D. M. (2015). Cosmic X-ray surveys of distant active galaxies. The demographics, physics, and ecology of growing supermassive black holes. Astron. Astrophys. Rev. 23:1. doi: 10.1007/s00159-014-0081-z

Brightman, M., and Nandra, K. (2011). An XMM-Newton spectral survey of $12 \mu \mathrm{m}$ selected galaxies - I. X-ray data. Month. Notices R. Astron. Soc. 413, 1206-1235. doi: 10.1111/j.1365-2966.2011.18207.x

Broos, P., Townsley, L., Getman, K., and Bauer, F. (2012). AE: ACIS Extract. Astrophysics Source Code Library.
We investigated the host galaxy properties of these quasars through their SFR, measured in the FIR band from SED fitting using Spitzer and Herschel data, and did not find any strong link between the amount of SF and the X-ray obscuration, possibly suggesting that the X-ray obscuration is mostly concentrated in the nuclear regions and does not depend on the presence of gas on larger scales.

We also visually classified the morphology of these quasars as disturbed or undisturbed using high-resolution HST data to identify signs of distortions/asymmetries in the galaxies. We find that a significant fraction $(\sim 40 \%)$ have disturbed morphologies, suggesting they have experienced a recent merger or interaction event. We find a larger fraction of sources with disturbed morphologies amongst the heavily-obscured quasars ( 53\%) rather than the unobscured/moderately-obscured ones ( $\sim 20 \%)$. Our results possibly support the SMBH-galaxy evolutionary scenario where the heavily-obscured quasars represent an earlier stage of evolution after the merger, while the unobscured quasars represent a later stage of the evolution, when the system has relaxed, the signs of interaction have already faded, and the nucleus becomes unobscured.

\section{AUTHOR CONTRIBUTIONS}

$\mathrm{AD}$ developed the concept, performed all the analyses and wrote the manuscript. DA initiated and helped developing the concept. FB provided the X-ray spectra. ED compiled the multiwavelength catalog, which was used for the SED analysis. DK and DM provided the optical HST images, used to classify the galaxy morphology. FS calculated the mean star-formation rates.

\section{FUNDING}

This research was supported by the UK Science and Technology Facilities Council (STFC, ST/L00075X/1); CONICYT-Chile (Basal-CATA PFB-06/2007, "EMBIGGEN" Anillo ACT1101, FONDECYT Regular 1141218); the Ministry of Economy, Development, and Tourism's Millennium Science Initiative through grant IC120009, awarded to The Millennium Institute of Astrophysics (MAS).

Broos, P. S., Townsley, L. K., Feigelson, E. D., Getman, K. V., Bauer, F. E., and Garmire, G. P. (2010). Innovations in the analysis of chandra-ACIS observations. Astrophys. J. 714, 1582-1605. doi: 10.1088/0004-637X/714/ 2/1582

Cisternas, M., Jahnke, K., Inskip, K. J., Kartaltepe, J., Koekemoer, A. M., Lisker, T., et al. (2011). The bulk of the black hole growth since $z \sim 1$ occurs in a secular universe: no major merger-AGN connection. Astrophys. J. 726:57. doi: 10.1088/0004-637X/726/2/57

Conselice, C. J., Bershady, M. A., Dickinson, M., and Papovich, C. (2003). A direct measurement of major galaxy mergers at $z \lesssim 3$. Astron. J. 126, 1183-1207. doi: $10.1086 / 377318$

Del Moro, A., Alexander, D. M., Bauer, F. E., Daddi, E., Kocevski, D. D., McIntosh, D. H., et al. (2016). Mid-infrared luminous quasars in the GOODS-Herschel fields: a large population of heavily obscured, Compton-thick quasars at $\mathrm{z} \approx 2$. Month. Notices R. Astron. Soc. 456, 2105-2125. doi: 10.1093/mnras/stv2748

Del Moro, A., Alexander, D. M., Mullaney, J. R., Daddi, E., Pannella, M., Bauer, F. E., et al. (2013). GOODS-Herschel: radio-excess signature of hidden 
AGN activity in distant star-forming galaxies. Astron. Astrophys. 549:A59. doi: 10.1051/0004-6361/201219880

Di Matteo, T., Springel, V., and Hernquist, L. (2005). Energy input from quasars regulates the growth and activity of black holes and their host galaxies. Nature 433, 604-607. doi: 10.1038/nature03335

Elbaz, D., Dickinson, M., Hwang, H. S., Díaz-Santos, T., Magdis, G., Magnelli, B., et al. (2011). GOODS-Herschel: an infrared main sequence for star-forming galaxies. Astron. Astrophys. 533:A119. doi: 10.1051/0004-6361/201117239

Ferrarese, L., and Merritt, D. (2000). A fundamental relation between supermassive black holes and their host galaxies. Astrophys. J. Lett. 539, L9-L12. doi: $10.1086 / 312838$

Fiore, F., Puccetti, S., Brusa, M., Salvato, M., Zamorani, G., Aldcroft, T., et al. (2009). Chasing highly obscured QSOs in the COSMOS field. Astrophys. J. 693, 447-462. doi: 10.1088/0004-637X/693/1/447

Gandhi, P., Horst, H., Smette, A., Hönig, S., Comastri, A., Gilli, R., et al. (2009). Resolving the mid-infrared cores of local Seyferts. Astron. Astrophys. 502, 457-472. doi: 10.1051/0004-6361/200811368

Gebhardt, K., Bender, R., Bower, G., Dressler, A., Faber, S. M., Filippenko, A. V., et al. (2000). A relationship between nuclear black hole mass and galaxy velocity dispersion. Astrophys. J. Lett. 539, L13-L16. doi: 10.1086/312840

Giavalisco, M., Ferguson, H. C., Koekemoer, A. M., Dickinson, M., Alexander, D. M., Bauer, F. E., et al. (2004). The great observatories origins deep survey: initial results from optical and near-infrared imaging. Astrophys. J. Lett. 600, L93-L98. doi: 10.1086/379232

Grogin, N. A., Kocevski, D. D., Faber, S. M., Ferguson, H. C., Koekemoer, A. M., Riess, A. G., et al. (2011). CANDELS: the cosmic assembly near-infrared deep extragalactic legacy survey. Astrophys. J. Suppl. 197:35. doi: 10.1088/0067-0049/197/2/35

Hopkins, P. F., Hernquist, L., Cox, T. J., Di Matteo, T., Robertson, B., and Springel, V. (2006). A unified, merger-driven model of the origin of starbursts, quasars, the cosmic X-ray background, supermassive black holes, and galaxy spheroids. Astrophys. J. Suppl. 163, 1-49. doi: 10.1086/499298

Kaplan, E. L., and Meier, P. (1958). Nonparametric estimation from incomplete observations. J. Am. Stat. Assoc. 53, 457-481. doi: 10.1080/01621459.1958.10501452

Kartaltepe, J. S., Sanders, D. B., Scoville, N. Z., Calzetti, D., Capak, P., Koekemoer, A., et al. (2007). Evolution of the frequency of luminous $\left(>=\mathrm{L}^{*}{ }_{V}\right)$ close galaxy pairs at $z<1.2$ in the COSMOS field. Astrophys. J. Suppl. 172, 320-328. doi: $10.1086 / 519953$

Kennicutt, R. C. Jr. (1998). Star formation in galaxies along the hubble sequence. Annu. Rev. Astron. Astrophys. 36, 189-232. doi: 10.1146/annurev.astro.3 6.1.189

Kocevski, D. D., Brightman, M., Nandra, K., Koekemoer, A. M., Salvato, M., Aird, J., et al. (2015). Are compton-thick AGNs the missing link between mergers and black hole growth? Astrophys. J. 814:104. doi: 10.1088/0004-637X/814/2/104

Kocevski, D. D., Faber, S. M., Mozena, M., Koekemoer, A. M., Nandra, K., Rangel, C., et al. (2012). CANDELS: constraining the AGN-Merger connection with host morphologies at $z \sim 2$. Astrophys. J. 744:148. doi: 10.1088/0004-637X/744/ $2 / 148$

Kormendy, J., and Ho, L. C. (2013). Coevolution (or not) of supermassive black holes and host galaxies. Annu. Rev. Astron. Astrophys. 51, 511-653. doi: 10.1146/annurev-astro-082708-101811

Kormendy, J., and Richstone, D. (1995). Inward bound-the search for supermassive black holes in galactic nuclei. Annu. Rev. Astron. Astrophys. 33:581.

Lotz, J. M., Jonsson, P., Cox, T. J., and Primack, J. R. (2010). The effect of mass ratio on the morphology and time-scales of disc galaxy mergers. Month. Notices $R$. Astron. Soc. 404, 575-589. doi: 10.1111/j.1365-2966.2010.16268.x
Luo, B., Bauer, F. E., Brandt, W. N., Alexander, D. M., Lehmer, B. D., Schneider, D. P., et al. (2008). The chandra deep field-south survey: 2 ms source catalogs. Astrophys. J. Suppl. 179, 19-36. doi: 10.1086/591248

Luo, B., Brandt, W. N., Xue, Y. Q., Lehmer, B., Alexander, D. M., Bauer, F. E., et al. (2017). The chandra deep field-south survey: $7 \mathrm{~ms}$ source catalogs. Astrophys. J. Suppl. 228:2. doi: 10.3847/1538-4365/228/1/2

Lutz, D., Maiolino, R., Spoon, H. W. W., and Moorwood, A. F. M. (2004). The relation between AGN hard X-ray emission and mid-infrared continuum from ISO spectra: scatter and unification aspects. Astron. Astrophys. 418, 465-473. doi: 10.1051/0004-6361:20035838

Madau, P., Ferguson, H. C., Dickinson, M. E., Giavalisco, M., Steidel, C. C., and Fruchter, A. (1996). High-redshift galaxies in the Hubble Deep Field: colour selection and star formation history to $\mathrm{z}^{\sim} 4$. Month. Notices R. Astron. Soc. 283, 1388-1404. doi: 10.1093/mnras/283.4.1388

Magorrian, J., Tremaine, S., Richstone, D., Bender, R., Bower, G., Dressler, A., et al. (1998). The demography of massive dark objects in galaxy centers. Astron. J. 115, 2285-2305. doi: 10.1086/300353

Mullaney, J. R., Alexander, D. M., Goulding, A. D., and Hickox, R. C. (2011). Defining the intrinsic AGN infrared spectral energy distribution and measuring its contribution to the infrared output of composite galaxies. Month. Notices R. Astron. Soc. 414, 1082-1110. doi: 10.1111/j.1365-2966.2011.18448.x

Ricci, C., Bauer, F. E., Treister, E., Schawinski, K., Privon, G. C., Blecha, L. et al. (2017). Growing supermassive black holes in the late stages of galaxy mergers are heavily obscured. Month. Notices R. Astron. Soc. 468, 1273-1299. doi: 10.1093/mnras/stx173

Rosario, D. J., Santini, P., Lutz, D., Shao, L., Maiolino, R., Alexander, D. M., et al. (2012). The mean star formation rate of X-ray selected active galaxies and its evolution from $\mathrm{z} \sim 2.5$ : results from PEP-Herschel. Astron. Astrophys. 545:A45. doi: 10.1051/0004-6361/201219258

Rovilos, E., Comastri, A., Gilli, R., Georgantopoulos, I., Ranalli, P., Vignali, C., et al. (2012). GOODS-Herschel: ultra-deep XMM-Newton observations reveal AGN/star-formation connection. Astron. Astrophys. 546:A58. doi: 10.1051/0004-6361/201218952

Salpeter, E. E. (1955). The luminosity function and stellar evolution. Astrophys. J. 121:161.

Stanley, F., Harrison, C. M., Alexander, D. M., Swinbank, A. M., Aird, J. A., Del Moro, A., et al. (2015). A remarkably flat relationship between the average star formation rate and AGN luminosity for distant X-ray AGN. Month. Notices $R$ Astron. Soc. 453, 591-604. doi: 10.1093/mnras/stv1678

Treister, E., and Urry, C. M. (2006). The evolution of obscuration in active galactic nuclei. Astrophys. J. Lett. 652, L79-L82. doi: 10.1086/510237

Xue, Y. Q., Luo, B., Brandt, W. N., Bauer, F. E., Lehmer, B. D., Broos, P. S., et al. (2011). The chandra deep field-south survey: $4 \mathrm{~ms}$ source catalogs. Astrophys. J. Suppl. 195:10. doi: 10.1088/0067-0049/195/1/10

Yaqoob, T. (1997). X-ray transmission in cold matter: nonrelativistic corrections for compton scattering. Astrophys. J. 479, 184-189. doi: 10.1086/303843

Conflict of Interest Statement: The authors declare that the research was conducted in the absence of any commercial or financial relationships that could be construed as a potential conflict of interest.

Copyright (C) 2018 Del Moro, Alexander, Bauer, Daddi, Kocevski, Stanley and McIntosh. This is an open-access article distributed under the terms of the Creative Commons Attribution License (CC BY). The use, distribution or reproduction in other forums is permitted, provided the original author(s) or licensor are credited and that the original publication in this journal is cited, in accordance with accepted academic practice. No use, distribution or reproduction is permitted which does not comply with these terms. 